\title{
Residents' Insights and Ideas about Screen- Sharing in Primary Care Clinics
}

\author{
Kathlyn E. Fletcher ${ }^{1}$ Onur Asan ${ }^{1}$ Jeanne Tyszka ${ }^{1}$ \\ ${ }^{1}$ Division of General Internal Medicine, Department of Medicine, \\ Center for Patient Care and Outcomes Research, Medical College of \\ Wisconsin and the Milwaukee VAMC, Milwaukee, Wisconsin, \\ United States \\ Address for correspondence Kathlyn E. Fletcher, MD, MA, Division of \\ Medicine, Department of Patient Safety and Quality, Medical College \\ of Wisconsin, Milwaukee, Wisconsin, United States
}

Appl Clin Inform 2017;8:1153-1158.

\section{Abstract}

Keywords

- electronic health records

- internal medicine

- internship and residency

- medical education

- patient-provider communication
Background Previous studies described attending physicians' perceptions of the benefits and downsides of having the electronic health record (EHR) in the room during a clinical encounter. The perspective of residents has received little attention.

Objective The goal of this project was to solicit internal medicine residents' perception of EHR use in primary care clinic visits. In this report, we focus on residents' perception of screen-sharing.

Methods We conducted qualitative, semistructured interviews of internal medicine residents. Interviews were audiorecorded, transcribed, and anonymized. Using a deductive approach to data analysis, we coded the transcripts to identify themes of interest.

Results We included 21 residents. We identified eight themes related to screen-sharing. We identified opportunities, facilitators and barriers, and outcomes related to screensharing. We conceptualized the outcomes, facilitators, and barriers as falling into four categories: structural, patient based, physician based, and interactional.

Conclusion Wider dissemination and adoption of curricula designed to teach residents how to incorporate EHR into the clinical encounter is needed. In addition, our study demonstrates the need to focus this training, at least in part, on screen-sharing and management of sensitive information disclosure.

\section{Background and Significance}

Widespread implementation of electronic health records (EHRs) in primary care has changed the dynamics of doctor-patient interactions, workflow of providers, and time spent on patient care and documentation. ${ }^{1,2}$ Studies report potential benefits and negative outcomes of EHR use in health care. ${ }^{3,4}$ One of the highly affected user groups is residents. Studies show that when an EHR is present, residents spend a substantial amount of time on e-documentation (both during official duty hours and after hours) and less time with their patients. ${ }^{5-7}$ One recent study showed that during outpatient encounters, residents looked at the computer screen $34 \%$ of the visit time and typed $14 \%$ of the visit time on average. ${ }^{8}$ Another study compared patients' perceptions of internal medicine attending versus resident physicians; the study found patients were more likely to feel the computer adversely affected the amount of time residents spent talking, looking at, and examining them. ${ }^{9}$

Although residents get training regarding the clinical features of the EHRs they use, there is a paucity of effective training on efficient use of EHRs during the patient visit. ${ }^{10,11}$ There is little literature addressing the necessity of teaching residents and medical students strategies to integrate the EHR into overall communication during patient visits. ${ }^{2,12}$ In fact, in a study done by the Alliance for Clinical Education (a consortium focused on medical student education), clerkship directors expressed concern about the effect of the EHR on studentpatient relationships, noting that there was a lack of good role modeling from faculty regarding how to do this well. ${ }^{13}$ received

April 9, 2017

accepted after revision

October 14, 2017
Copyright @ 2017 Schattauer
DOI https://doi.org/ 10.4338/ACI-17-0057-RA. ISSN 1869-0327. 
Some literature has addressed this gap by suggesting best practices for using EHRs during visits to minimize the potential negative impact on doctor-patient communication. ${ }^{2,12,14,15}$ One way is by sharing the computer screen; this can engage patients more readily with their own health information during the visit. ${ }^{16,17}$ While some studies report potential benefits when providers share the screen, ${ }^{14,16}$ there is a need for additional studies on screen-sharing in general. Including residents' perceptions would be of value because they are underrepresented in published literature on this topic, and the opportunity to understand their viewpoints before they develop routines around EHR use could be very helpful.

\section{Objective}

The purpose of this study was to capture the perception of internal medicine residents on exam room EHR use, more specifically their views on facilitators and barriers surrounding screen-sharing, and advantages/disadvantages screen-sharing affords to patient education. We chose to focus on residents because they have not yet developed and become ingrained in a practice style, and so training could still theoretically be beneficial. To guide development of such training, residents' own perceptions needed to be understood.

\section{Methods}

\section{Overview}

This project was part of a larger study in which we explored the perceptions of residents and their patients surrounding EHR use in outpatient internal medicine clinics at the Medical College of Wisconsin. Here, we are reporting on residents' perceptions of screen-sharing; we reported patients' perceptions previously. ${ }^{18}$ The Institutional Review Board at the Medical College of Wisconsin approved this study.

\section{Study Setting and Participants}

We conducted semistructured interviews of internal medicine residents during a 4-month period from September to December 2014. We announced details about the study and sent follow-up email invitations to 125 residents at an urban academic medical college. Clinic assignments include urban, suburban, and Veterans Affairs settings. The inclusion criterion was continuity clinic assignment using EHRs for at least 4 months. We expected to reach data saturation (the point at which no new themes are emerging from the interviews ${ }^{19}$ ) at $n=20$, a small sample size that is standard in homogenous purposive samples. ${ }^{10,20}$ Twenty-four residents responded to the invitation, with 21 completed interviews. We suspended active recruitment when we reached data saturation at 18 participants, but continued to speak to residents who had interviews scheduled. J.T. or O.A. (i.e., the study team members not associated with the residency program) met each participant in a private room to explain the study, obtain written consent, and conduct the interview. We offered a $\$ 30$ gift card as an incentive to all residents.

\section{Data Collection}

We collected data through interviews and demographic surveys. We employed a semistructured interview guide, with seven questions and probes (see - Supplementary Table 1). Questions, derived from conversations with attending physicians and one author's previous studies, ${ }^{14,15}$ centered on the resident's communication style, training, screen-sharing behavior, and the perceived advantages/disadvantages of screensharing; additionally, we asked residents to discuss the work system facilitators/barriers surrounding screen-sharing. We based the facilitator/barrier questions on the Systems Engineering in Patient Safety model (SEIPS). ${ }^{21}$ J.T. conducted pilot interviews with two chief residents (not included in the analysis) as we developed the guide to ensure the questions and probes were understandable and suitable; we then used an iterative process during interviews to allow for the exploration of unexpected topics. Interviews were audiorecorded, transcribed, and anonymized. Interview length ranged from 23 to 40 minutes, with a mean of 32 minutes. The interviewer (J.T.) was trained by K.E.F. and A.O., who both have qualitative research experience.

\section{Analysis}

When the first four interview recordings were transcribed, we began the process of coding. We applied deductive content analysis approach to analyze the interview data. ${ }^{22,23}$ We used NVivo 10, qualitative analysis software, to analyze the data in the interview transcripts. The study team reviewed these transcripts and devised a provisional coding scheme using deductive analysis; each member of the study team then coded one transcript using this scheme. Concurrently with further data collection, the study team met weekly to apply and revise the scheme to additional transcripts. We developed a thematic codebook with 18 defined codes, 8 of which relate to screensharing (see - Supplementary Table 2). Discrepancies in coding were discussed until consensus was reached. After all data had been collected and initial coding was completed, the research assistant (J.T.) did second cycle coding, or condensed analysis, within each coding category. Second cycle coding was discussed by the entire team in subsequent meetings. Two study team members independently coded two of the transcripts and compared their coding to calculate an agreement score which was $89 \%$.

\section{Results}

Participant characteristics can be found in -Table 1. In our qualitative analysis, we found eight codes related to screensharing (see -supplementary Table 2). Within the text assigned to these codes, we identified opportunities, work system factors that aided screen-sharing (facilitators) or hindered screen-sharing (barriers), and perceived advantages/disadvantages to screen-sharing. We conceptualized the advantages, disadvantages, facilitators, and barriers as falling into four categories: structural, patient based, physician based, and interactional (-Table 2). "Structural" refers to the SEIPS work system components, "patient based" refers to the resident's perception of factors that involve patients, 
Table 1 Resident demographics

\begin{tabular}{|c|c|}
\hline & Participant demographic \\
\hline \multirow[t]{2}{*}{ Gender } & Male $=12(57 \%)$ \\
\hline & Female $=9(43 \%)$ \\
\hline \multirow[t]{3}{*}{ PGY } & PGY1 = 6 (29\%) \\
\hline & PGY2 = 7 (33\%) \\
\hline & PGY3 = $8(38 \%)$ \\
\hline \multirow[t]{2}{*}{ Race } & Asian $=9(43 \%)$ \\
\hline & White $=12(57 \%)$ \\
\hline \multirow[t]{2}{*}{ Age } & Range: 26-35 \\
\hline & Mean: 28.7 \\
\hline \multirow[t]{3}{*}{ Comfort using EHR } & Very comfortable $=8(38 \%)$ \\
\hline & Comfortable $=9(43 \%)$ \\
\hline & Varies $=4(19 \%)$ \\
\hline \multirow{2}{*}{$\begin{array}{l}\text { Previous experience } \\
\text { with EHR }\end{array}$} & Yes $=20(95 \%)$ \\
\hline & No $=1(5 \%)$ \\
\hline \multirow[t]{3}{*}{ Clinic setting } & $\begin{array}{l}\text { Regional medical center } \\
\text { (urban) }=10(48 \%)\end{array}$ \\
\hline & Suburban = 7 (33\%) \\
\hline & $V A=4(19 \%)$ \\
\hline
\end{tabular}

Abbreviations: EHR, electronic health record; PGY, postgraduate year; $V A$, veterans affairs.

"physician based" refers to factors that impact the physician her/himself, and "interactional" refers to factors arising from the interaction of physician and patient.

\section{Opportunities for Screen-Sharing}

Residents reported many opportunities for screen-sharing within the EHR, including showing the patients trends such as laboratories, blood pressures, and weights. One resident described the reinforcement of screen-sharing for his verbalization: “'your cholesterol is going up'; seeing it makes it more real and more, um, hopefully improves compliance... hopefully it does." They also described sharing the screen to go over images and medication lists and to review postcare instructions and consent forms. In addition, the residents also shared the screen when using risk calculators, finding traveler information, and showing patients instructions, such as exercises for physical therapy. "...really nice online tool that shows what their risk is today and what it could be if they did like, style changes."

\section{Screen-Sharing Advantages/Disadvantages}

Advantages to screen-sharing fell into patient-based, physician-based, and interactional advantages. The patient-based advantages included the ability to reassure patients, encourage them to take ownership of their health care, and possibly even to improve their outcomes. Residents also noted that screen-sharing allowed the patients to double check the accuracy of the computerized medication list. Some residents thought that sharing the screen may improve understanding of lower literacy patients, as one resident said, "...actually for patients with lower health literacy, I might share the screen a little more often with them. Patients with high health literacy, it's very easy to talk through things and concepts that they're familiar with, but I think it's probably the patients with the lowest health literacy that I feel have felt the most use - or I feel like we've made a better connection after I share the screen with them and they see what I'm saying." Residents also noted that seeing serious results in the EHR could also be a disadvantage.

The main physician-based advantage occurred when the resident had the opportunity to synthesize multiple providers' recommendations while screen-sharing with patients. That is, by allowing the patient to see notes written by other providers, the primary care physician was able to justify plans that were consistent with care provided across many appointments. A physician-based disadvantage is that screen-sharing takes more time.

Interactional advantages included making the visit more collaborative and increasing opportunities for shared decision making. The residents described educational opportunities when they were screen-sharing, for example, providing

Table 2 Examples of advantages/disadvantages and facilitators/barriers of screen-sharing

\begin{tabular}{|c|c|c|c|c|}
\hline & Structural & Patient based & Physician based & Interactional \\
\hline $\begin{array}{l}\text { Advantages/ } \\
\text { Disadvantages }\end{array}$ & None & $\begin{array}{l}\text { Advantage } \\
\text { Patients can double check } \\
\text { accuracy of medication list } \\
\text { Disadvantage } \\
\text { Patients may feel } \\
\text { distressed about seeing } \\
\text { results }\end{array}$ & $\begin{array}{l}\text { Advantage } \\
\text { Residents can synthesize } \\
\text { information from several } \\
\text { doctors } \\
\text { Disadvantage } \\
\text { Takes more time }\end{array}$ & $\begin{array}{l}\text { Advantage } \\
\text { Residents can educate } \\
\text { patients about health } \\
\text { trends } \\
\text { Disadvantage } \\
\text { Difficult to explain why } \\
\text { sensitive information is } \\
\text { written in the record }\end{array}$ \\
\hline $\begin{array}{l}\text { Facilitators/ } \\
\text { Barriers }\end{array}$ & $\begin{array}{l}\text { Facilitators } \\
\text { Room layout; } \\
\text { computer screens } \\
\text { that swivel } \\
\text { Barriers } \\
\text { Slow computers }\end{array}$ & $\begin{array}{l}\text { Facilitators } \\
\text { Having a family member } \\
\text { present who wanted to } \\
\text { see information on the } \\
\text { screen } \\
\text { Barriers } \\
\text { Patients with poor vision }\end{array}$ & $\begin{array}{l}\text { Facilitators } \\
\text { Resident skills at } \\
\text { navigating EHR } \\
\text { Barriers } \\
\text { Not having enough time }\end{array}$ & None \\
\hline
\end{tabular}

Abbreviation: EHR, electronic health record. 
evidence of trends, as this resident explained, "Advantages from the standpoint of echoing what you're trying to tell a patient, and I think it gives more, um, factual evidence. I think sometimes we run into the issues of, 'well doc, I felt that I lost weight' and then you can show them the actual numbers and say, yes or no..." They also described turning the computer into an engagement device rather than a separator. "The advantage to screen-sharing] is to engage the patient and to, um, have the computer monitor not look like it's a barrier between us, but as a tool that we're both using." Another resident elaborated on this point: “...the definite advantage and the reason that I do it is to try to get the patient to take ownership of their health and, you know, get buy in for what we're - what we're trying to do, it's my - it's my hope that if they see their - you know, see their blood pressure trend and they can - I can show them, well look, here we started this medication, this is what happened to it..." Sharing the screen allowed the residents to feel that they were not dividing their attention between the computer and the patient. Residents also reported feeling that screen-sharing facilitated trust and transparency: "Especially if they already have, you know, inherent mistrust of - of healthcare providers, um, but in general, you - I think sharing of the screen just creates an environment of transparency, um, and shows that you're not - you're not doing anything nefarious behind your screen."

Residents identified several interactional disadvantages to screen-sharing. Many worried about how to manage the sensitive information that patients conveyed to them. One resident explained, "The only disadvantage I can see is ... if there's sensitive information which maybe the patient is willing to tell you but doesn't necessarily want put into the medical record." The resident wondered if the patients realized that confidential communication was documented in the written record. Additionally, the residents worried about patients seeing terms written in the record that might be misunderstood or seem offensive, as this resident noted, “...if, you know, my exam I'm saying, abdomen obese... ...those sorts of things could potentially be offensive." They noted that writing a differential diagnosis might include information that was unnecessarily alarming to the patient. Residents also noted that typing can be awkward and can take focus away from the patient. One resident even stated that screen-sharing seemed like delivering a PowerPoint lecture.

\section{Screen-Sharing Facilitators/Barriers}

The residents identified several structural factors that facilitate screen-sharing including the layout of the room (e.g., being able to accommodate a wheelchair for screen-sharing), having computer screens that swivel or other technology that faced the patient, having computers that were quick and did not freeze, and aspects of the EHR itself. Aspects of the EHR that facilitated screen-sharing were note templates that walked the provider through the visit, flowsheets, and easyto-follow colors/fonts. They also noted that being able to open Web sites during the visit facilitated screen-sharing.

Patient-based factors that facilitated screen-sharing included having high health literacy, and being younger. Dementia, poor vision, and being nonfluent in English were barriers. Being accompanied by a family member who also wanted to see the information could act as a facilitator or a barrier to screen-sharing: "So if you have family members who are more health literate than the patient themselves, or who are a caregiver for the patient and they would benefit from understanding or visually, you know, appreciating what is - has transpired, then it's absolutely a facilitator. Because then somebody gets it and somebody's there and can watch and can really take the information away from that visit. If there's a family member who, instead is very anxious and does not understand the situation and would probably take that situation home and make it a - just, I don't know, a nightmare for the patient...." Residents felt that some patients were simply not interested in seeing their data.

Physician-based barriers to screen-sharing included not having enough time, as this resident described, “...ifI'm running late, obviously it might be difficult for me to spend the appropriate amount of time showing results or digging into EHR with the patient." Another barrier was not having well-developed EHR skills and experience: "II] am not the most savvy EPIC user, um, so for example, um, I probably don't show, um, charts of data or graphs of data because I don't know how to readily bring up graphs of data ... And I'm probably not the fastest type."

\section{Strategies for Screen-Sharing}

Residents reported several strategies they used for screensharing. To begin, some residents assess patient's level of interest in screen-sharing, and make their decision about sharing according to the patient's desire. Some described verbally inviting the patients to view the screen with them: "I might say, hey, check this out, look at your - look at how you're doing, your - your weight is down 15 pounds, that's terrific, keep up the good work..." The residents adjust the structural aspects of the room/computer to facilitate screensharing. Examples included moving the physician's chair closer to the patient and tilting or swiveling the monitor toward the patient. One resident said, "I usually pull my chair back and turn the screen so they can, obviously see it better, but also to kind of let them know ... this is their information." During screen-sharing, the residents reported strategies such as using the mouse to point to things, using graphing functions, and showing the patient how they navigate through the EHR. One issue that was discussed frequently was how to handle sensitive information. Residents were particularly worried about having the patients see that they were typing sensitive information. Strategies that they used for this situation included turning the screen away from the patients during all typing, turning the screen away during typing about sensitive information, and not typing sensitive information while with the patient.

\section{Discussion}

In this study, residents identified advantages/disadvantages, facilitators/barriers to screen-sharing with patients during primary care appointments; these could be categorized as structural, patient-based, physician-based, or interactional. Residents also discussed opportunities and strategies for accomplishing screen-sharing during such visits. 
In this study, residents described that screen-sharing made the computer an instrument of engagement rather than an instrument of separation. This finding is encouraging, given that there has been concern that having a computer in the room detracts from the doctor-patient relationship. ${ }^{16,24}$ This is not to say that the computer always acts to enhance communication, rather that when the decision is made to share the screen, many opportunities exist for enhancing rather than worsening communication.

The most extensive curriculum that we identified is the Improving Patient-Centered Technology Use (iPaCT) program. ${ }^{25}$ The iPaCT program comes with educational materials and tools such as pocket cards with 10 recommendations for making EHR use more patient centered. ${ }^{25}$ Many of their recommendations were corroborated by our residents, such as using charting tools for education and the importance of sharing the screen with patients. In our study, the residents identified several opportunities for screen-sharing that could be highlighted in future curricula.

The iPaCT curriculum also addresses our residents' concern about documenting sensitive patient information. The iPaCT suggestion is to "Nix the screen" during sensitive conversations. Perhaps the most reasonable strategy for resident and faculty physicians alike is to disengage from the computer during sensitive conversations, and to add this documentation to the chart after the visit has concluded. This recommendation does not completely address our residents' concerns, though. Specifically, if physicians share the screen to increase transparency, patients may see sensitive or potentially offensive information that was documented previously. Our study does not offer any particular strategy for managing that situation, but rather highlights that more research is needed to understand how to best manage this from the patients' perspective and from expert physician communicators.

In this study, residents had helpful ideas about how to facilitate screen-sharing in the primary care setting. In a previous study that included residents, they expressed concern about learning how to use EHRs at the same time that they were developing their own communication styles, ${ }^{26}$ suggesting that we do indeed need guidance from residents about the issues that they are facing with their own professional development, especially with respect to patient-centered EHR use. Our data also suggest that suboptimal EHR skills and feeling pressed for time are both potential barriers to screen-sharing for residents. Improving training in navigating the EHR with and without patients present may be a needed adjunct to communication curricula like iPaCT. Of course, many suggestions from residents will be applicable to faculty and practicing physicians as well. Residents are well positioned to make these recommendations because they are on the frontlines, they are looking at these issues with new eyes (compared with faculty), and because many of their generation are technically savvy. We must listen and use their input because we need more internal medicine residents in primary care. If we can minimize the frustration associated with having the computer in the examination room, then perhaps we will have to remove one of the barriers.
This study has several limitations. First, this was a singlesite study, which may limit generalizability. All the participants were internal medicine residents, and so these principles may not apply to other specialties. Participation was voluntary, and the residents who chose to volunteer may have been those most comfortable with the EHR, which may also limit generalizability. The qualitative nature of the study does not allow us to draw conclusions about how common these findings are or how strongly they are felt.

\section{Conclusion}

In summary, internal medicine residents identified opportunities for screen-sharing in their own primary care clinics. In general, they saw potential for screen-sharing to improve communication and increase understanding with their patients. We recommend structural improvements such as having computers that swivel and software that makes it easier to see for patients with low vision. We also recommend widespread adoption of curricula to enhance patient-centered EHR use, focusing special attention on how to share the screen for educational purposes and how to manage sensitive information within the EHR. This last point probably requires additional input from patients. Future quality improvement work should focus on how to document and share sensitive/potentially offensive information with patients. Future research should link patient and physician perspectives on interventions designed to improve EHR-based communication and education. Outcomes of interest include patient knowledge about health status, patient empowerment, patient adherence, and physician burn-out and time spent on each patient encounter (including charting before and after the visit).

\section{Clinical Relevance Statement}

The results of this study suggest that primary care visits offer many opportunities for screen-sharing between physicians and patients. Internal medicine residents view screen-sharing as an opportunity to improve communication with their patients. Training for residents and students should focus on anticipating and planning for difficult situations that may arise with screen-sharing as well as strategies for maximizing its use.

\section{Multiple Choice Questions}

1. Which of the following is an opportunity for screensharing during a primary care visit?

A. When you are reviewing laboratory trends

B. When you are conducting the physical examination

C. When the nurse is injecting vaccines

D. When you are not in the room with the patient

Correct Answer: The correct answer is A. Reviewing laboratory trends is an excellent opportunity to invite patients to see how their disease control has been. Residents discussed showing patients' trends in blood pressure and weight as well as laboratory values. Residents felt that 
this offered more evidence to the patients about their health status. Sharing these trends also invited patients to take ownership of their health.

2. Which of the following is NOT a strategy for screensharing with patients during primary care visits.

A. Swiveling the computer so that patients can see it

B. Inviting the patient to look at the screen

C. Using a scribe during the visit

D. Repositioning chair so that both patient and physician can visualize screen

Correct Answer: The correct answer is C. Residents identified several strategies that they use to facilitate screensharing during primary care appointments, including swiveling the computer for easier patient viewing, moving their own chairs to sit next to patients, and actually inviting the patients to look at the screen. Using a scribe was not one of the strategies described by our residents. Depending on how a scribe is deployed in the clinical setting, the scribe may actually be the person using the computer which would not facilitate the physician and patient sharing the screen.

\section{Protection of Human and Animal Subjects}

The study was performed in compliance with the World Medical Association Declaration of Helsinki on Ethical Principles for Medical Research Involving Human Subjects, and was reviewed by the Medical College of Wisconsin Institutional Review Board.

\section{Conflict of Interest}

None.

\section{Acknowledgments}

The authors gratefully acknowledge the physicians and patients who agreed to participate in this study, as well as the financial support provided by the Clinical and Translational Science Award (CTSA) program of the National Center for Research Resources and the National Center for Advancing Translational Sciences (grant: UL1TR000055) and financial support and infrastructure provided by the Clinical and Translational Science Institute of Southeast Wisconsin through the Medical College Physicians Group.

\section{References}

1 Ventres W. Electronic health records: upsides, downsides, and inside-outsides on the way toward their use in clinical practice. Teach Learn Med 2013;25(04):366-368

2 Duke P, Frankel RM, Reis S. How to integrate the electronic health record and patient-centered communication into the medical visit: a skills-based approach. Teach Learn Med 2013;25(04):358-365

3 Lown BA, Rodriguez D. Commentary: lost in translation? How electronic health records structure communication, relationships, and meaning. Acad Med 2012;87(04):392-394

4 Asan OD Smith P, Montague E. More screen time, less face time implications for EHR design. J Eval Clin Pract 2014;20(06):896-901
5 Oxentenko AS, Manohar CU, McCoy CP, et al. Internal medicine residents' computer use in the inpatient setting. J Grad Med Educ 2012;4(04):529-532

6 Gilleland M, Komis K, Chawla S, Fernandez S, Fishman M, Adams M. Resident duty hours in the outpatient electronic health record era: inaccuracies and implications. J Grad Med Educ 2014;6(01):151-154

7 Chen L, Guo U, Illipparambil LC, et al. Racing against the clock: internal medicine residents' time spent on electronic health records. J Grad Med Educ 2016;8(01):39-44

8 Asan O, Kushner K, Montague E. Exploring residents' interactions with electronic health records in primary care encounters. Fam Med 2015;47(09):722-726

9 Rouf E, Whittle J, Lu N, Schwartz MD. Computers in the exam room: differences in physician-patient interaction may be due to physician experience. J Gen Intern Med 2007;22(01):43-48

10 Morrow JB, Dobbie AE, Jenkins C, Long R, Mihalic A, Wagner J. Firstyear medical students can demonstrate EHR-specific communication skills: a control-group study. Fam Med 2009;41(01):28-33

11 Heiman HL, Rasminsky S, Bierman JA, et al. Medical students' observations, practices, and attitudes regarding electronic health record documentation. Teach Learn Med 2014;26(01):49-55

12 Graham-Jones P, Jain SH, Friedman CP, Marcotte L, Blumenthal D. The need to incorporate health information technology into physicians' education and professional development. Health Aff (Millwood) 2012;31(03):481-487

13 Hammoud MM, Margo K, Christner JG, Fisher J, Fischer SH, Pangaro LN. Opportunities and challenges in integrating electronic health records into undergraduate medical education: a national survey of clerkship directors. Teach Learn Med 2012;24(03):219-224

14 Asan O. Providers' perceived facilitators and barriers to EHR screen sharing in outpatient settings. Appl Ergon 2017;58:301-307

15 Asan O, Carayon P, Beasley JW, Montague E. Work system factors influencing physicians' screen sharing behaviors in primary care encounters. Int J Med Inform 2015;84(10):791-798

16 White A, Danis M. Enhancing patient-centered communication and collaboration by using the electronic health record in the examination room. JAMA 2013;309(22):2327-2328

17 Saleem JJ, Flanagan ME, Russ AL, et al. You and me and the computer makes three: variations in exam room use of the electronic health record. J Am Med Inform Assoc 2014;21(e1):e147-e151

18 Asan O, Tyszka J, Fletcher KE. Capturing the patients' voices: planning for patient-centered electronic health record use. Int J Med Inform 2016;95:1-7

19 Creswell JW. Qualitative Inquiry and Research Design: Choosing among Five Traditions. Thousand Oaks, CA: SAGE; 1998

20 Robson C. Real World Research: Oxford: Blackwell; 2002

21 Carayon P, Schoofs Hundt A, Karsh BT, et al. Work system design for patient safety: the SEIPS model. Qual Saf Health Care 2006;15 (Suppl 1):i50-i58

22 Elo S, Kyngäs $\mathrm{H}$. The qualitative content analysis process. J Adv Nurs 2008;62(01):107-115

23 Forman J, Damschroder L. Qualitative content analysis. Adv Bioeth 2007;11:39-62

24 Crampton NH, Reis S, Shachak A. Computers in the clinical encounter: a scoping review and thematic analysis. J Am Med Inform Assoc 2016;23(03):654-665

25 Alkureishi MLW, Farnan J, Arora V. Breaking away from the iPatient to care for the real patient: implementing a patientcentered EMR use curriculum. MedEdPORTAL Publications: Association of American Medical Colleges; 2014

26 Ventres W, Kooienga S, Vuckovic N, Marlin R, Nygren P, Stewart V. Physicians, patients, and the electronic health record: an ethnographic analysis. Ann Fam Med 2006;4(02):124-131 